Supporting Information

\title{
Infrared-radiation-enhanced Nanofiber Membrane for Sky Radiative Cooling of the Human Body
}

Runcai Xiao, ${ }^{\dagger}$ Chengyi Hou, ${ }^{*}+\uparrow$ Weifeng Yang, ${ }^{\ddagger}$ Yun Su, Yaogang Li,§ Qinghong Zhang,\$ Peng Gao," Hongzhi Wang",

tKey Laboratory of High Performance Fibers \& Products, Ministry of Education, Donghua University, Shanghai 201620, P. R. China.

‡State Key Laboratory for Modification of Chemical Fibers and Polymer Materials, College of Materials Science and Engineering, Donghua University, Shanghai 201620, P. R. China.

'College of Fashion and Design, Donghua University, Shanghai 200051, P. R. China.

\$Engineering Research Center of Advanced Glasses Manufacturing Technology, Ministry of Education, Donghua University, Shanghai 201620, P. R. China.

"Tianjin Institute of Power Sources, Tianjin 300384, P. R. China.

*Corresponding authors emails: hcy@dhu.edu.cn and wanghz@dhu.edu.cn 


\section{Supplementary Notes}

Note S1. Thermal and moisture resistance test methods and calculation formulas for textiles.

Thermal resistance test: Sweating guarded hotplate (SGHP, Northwest Testing Technology Corporation, US) consists of three independantly controlled parts: test plate, guard ring and lower guard is used to measure the thermal resistance test. To simulate human bady skin and its surrounding environment, test plate and guard ring are set to the same temperature $\left(35^{\circ} \mathrm{C}\right)$, which can make sure test plate transfer heat upward to the environment $\left(25^{\circ} \mathrm{C}\right)$ through the textiles (Fig. S5). The heat energy (Q) required to maintain the set temperature of each part is measured. Thermal resistance calculation formula is expressed as:

$$
\boldsymbol{R}_{c t}=\frac{T_{\text {skin }}-\boldsymbol{T}_{e n v}}{\boldsymbol{Q}_{/ \mathrm{A}}}
$$

where $\boldsymbol{T}_{\text {skin }}$ is the temperature of test plate $\left({ }^{\circ} \mathrm{C}\right) ; \boldsymbol{T}_{\boldsymbol{e n v}}$ is the surrounding temperature; $\boldsymbol{Q} / \boldsymbol{A}$ is the test plate heat flow $\left(\mathrm{W} / \mathrm{m}^{2}\right)$.

Moisture resistance test: Moisture resistance test apparatus is only added a water barrier film between test plate and textiles, such as fiberglass, compared to thermal resistance test apparatus. The water barrier film allows water vapor pass through while liquid water isn't. Moisture resistance can be calculated by measuring the water vapor pressure above and below the textiles. The calculation formula is expressed as:

$$
\begin{gathered}
\boldsymbol{R}_{\text {et }}=\frac{P_{\text {skin }}-P_{\text {env }}}{Q / A} \\
P_{\text {env }}=\mathbf{R H} \cdot 133.3 \cdot 10^{\left[8.10765-\left(\frac{1750.29}{235+T_{e n v}}\right)\right]} \\
P_{\text {skin }}=133.3 \cdot 10^{\left[8.10765-\left(\frac{1750.29}{235+T_{\text {env }}}\right)\right]}
\end{gathered}
$$


where $\boldsymbol{P}_{\text {skin }}$ is the water vapor pressure on the test plate $(\mathrm{Pa}) ; \boldsymbol{P}_{\text {env }}$ is the ambient environment water vapor pressure $(\mathrm{Pa}) ; \boldsymbol{Q} / \boldsymbol{A}$ is the test plate heat flow $\left(\mathrm{W} / \mathrm{m}^{2}\right)$; $\mathrm{RH}$ is environment relative humidity $(\%)$.

Note S2. The evaluated cost of $\mathrm{PA} 6 / \mathrm{SiO}_{2} \mathrm{NFM}$, Commercial PA6 fabric and Commercial moisture wicking textile

The PA6/SiO 2 NFM $\left(20 * 20 \mathrm{~cm}^{2}\right.$, Electrospinning concentration is $20 \%$, Silica content is 20\%) need 0.2g PA6 pellets (about $0.0052 \mathrm{RMB}$ yuan), $0.8 \mathrm{~g}$ (about 0.65 $\mathrm{mL}$ ) Formic acid (about 0.3 RMB yuan) and $0.04 \mathrm{~g}$ Silica that needs $0.35 \mathrm{~mL}$ TEOS (about $0.056 \mathrm{RMB}$ yuan), $4.5 \mathrm{~mL}$ Ethanol (about $0.078 \mathrm{RMB}$ yuan) and $3 \mathrm{~mL}$ Ammonia solution (about 0.066 RMB yuan).

The total cost: $\mathrm{PA} 6 / \mathrm{SiO}_{2} \mathrm{NFM}: 0.5052 \mathrm{RMB}$ yuan $/ 400 \mathrm{~cm}^{2}=12.63 \mathrm{RMB}$ yuan $/ \mathrm{m}^{2}=$ $1.7714 \$ / \mathrm{m}^{2}$; Commercial PA6 fabric (Crystal shine red, China): $15 \mathrm{RMB}$ yuan $/ \mathrm{m}^{2}=$ $2.1038 \$ / \mathrm{m}^{2}$; Commercial moisture wicking textile (Camel, China): 39 RMB yuan per T-shirt (about $\left.1 \mathrm{~m}^{2}\right)=5.47 \$ / \mathrm{m}^{2}$ 


\section{Supplementary Table}

Table S1. The cost, model and specification of main raw materials and chemical reagent used for preparing the PA6/SiO2 NFM

\begin{tabular}{|c|c|c|}
\hline $\begin{array}{l}\text { Raw materials or } \\
\text { chemical reagent }\end{array}$ & Model and specification & cost \\
\hline PA6 pellets & $\begin{array}{c}\mathrm{Mw}=6.0 \times 10^{4}, \text { Arkema }, \\
\text { France }\end{array}$ & 26.43 RMB yuan/kg \\
\hline Formic acid & $\begin{array}{c}\text { 99\%, F112038-500 ml, } \\
\text { Shanghai Aladdin } \\
\text { Biochemical Technology } \\
\text { Co., Ltd }\end{array}$ & $229 \mathrm{RMB}$ yuan/500 mL \\
\hline TEOS & $\begin{array}{l}>99 \% \text { (GC), T110596- } \\
500 \text { ml, Shanghai Aladdin } \\
\text { Biochemical Technology } \\
\text { Co., Ltd }\end{array}$ & $80 \mathrm{RMB}$ yuan/500 mL \\
\hline Ethanol & $\begin{array}{c}\text { 95\%, AR, } 5 \text { L, Sinopharm } \\
\text { Chemical Reagent Co., } \\
\text { Ltd. }\end{array}$ & 87 RMB yuan/5 L \\
\hline Ammonia solution & $\begin{array}{c}25.0 \sim 28.0 \%, \text { GR, } 500 \\
\text { mL, Sinopharm Chemical } \\
\text { Reagent Co., Ltd. }\end{array}$ & $11 \mathrm{RMB}$ yuan/500 mL \\
\hline
\end{tabular}




\section{Supplementary Figures}
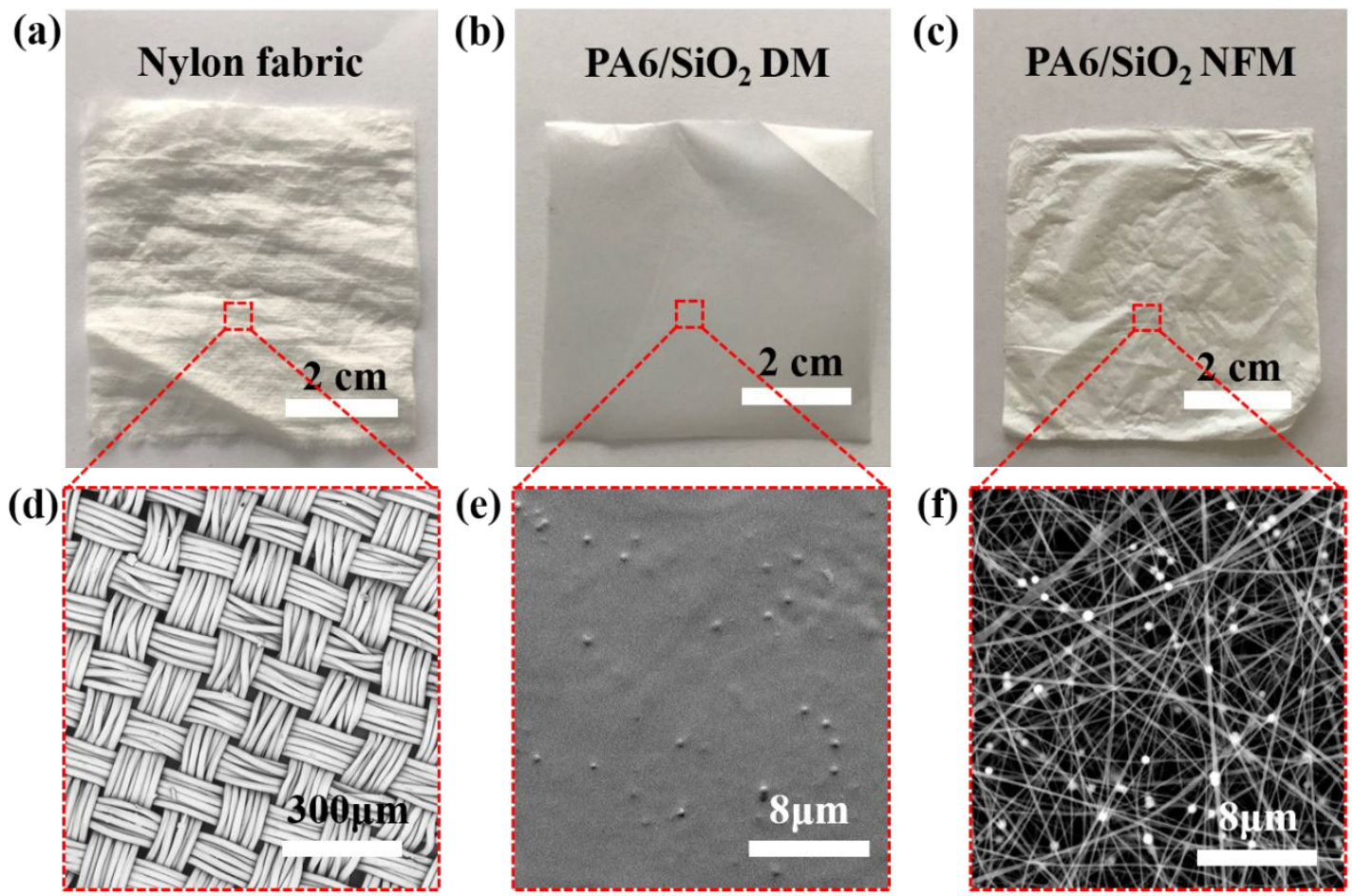

(e)
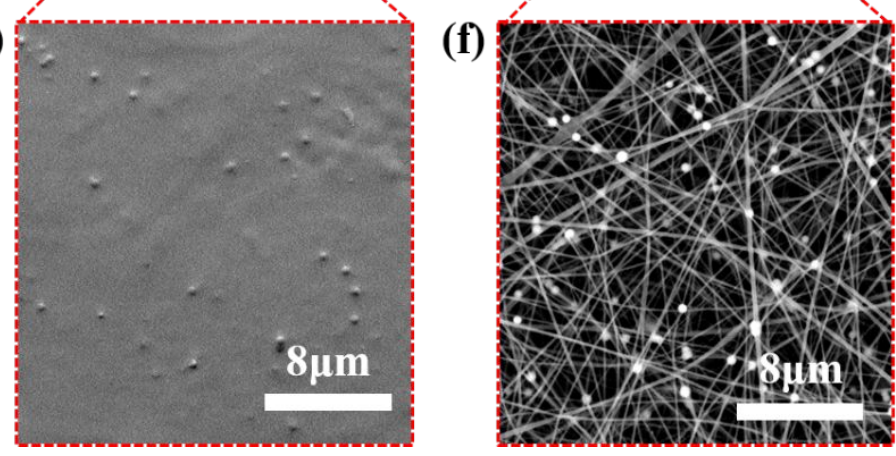

Figure S1. Photos of a) nylon fabric, b) $\mathrm{PA} 6 / \mathrm{SiO}_{2} \mathrm{DM}$ and c) $\mathrm{PA} / \mathrm{SiO}_{2} \mathrm{NFM}$; SEM image of d) nylon fabric, e) $\mathrm{PA} 6 / \mathrm{SiO}_{2} \mathrm{DM}$ and f) $\mathrm{PA} 6 / \mathrm{SiO}_{2} \mathrm{NFM}$. 
$\Longrightarrow \quad$ Solar power $(0.15-4 \mu \mathrm{m})$

$\longrightarrow \quad$ IR radiation

Air convection

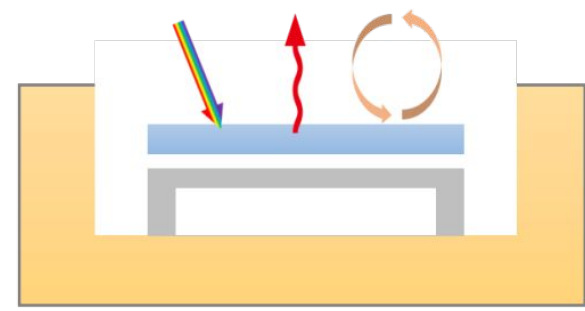

Remove convection barrier (Polyethylene film)

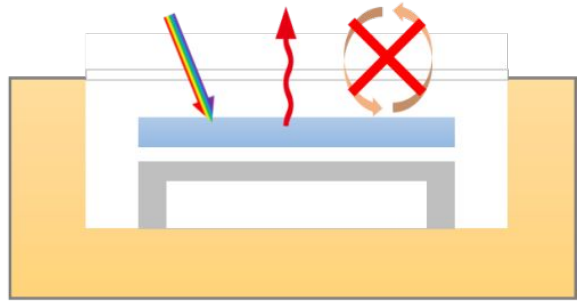

Put back convection barrier (Polyethylene film)

Figure S2. Schematic of thermal convection barrier (Polyethylene film) was first removed and then put back. In this process, the only change is that air convection is blocked. 
(a)

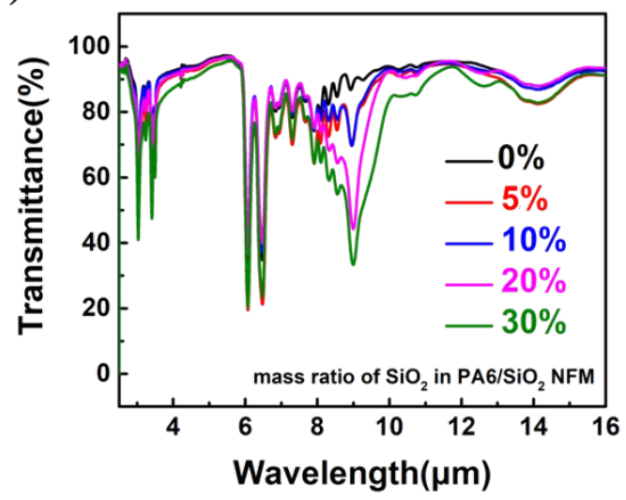

(b)

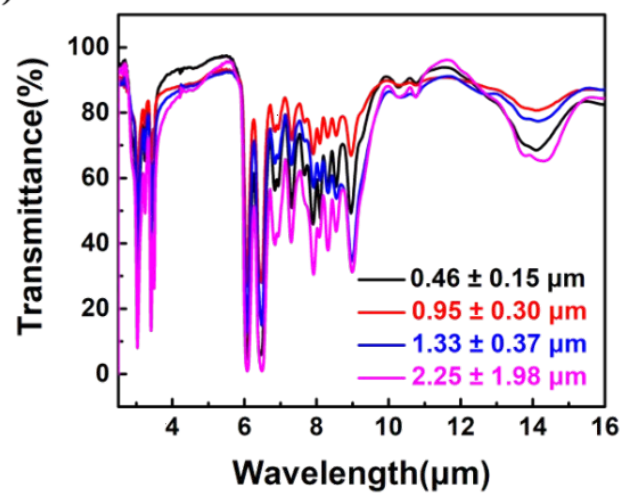

(c) $-18 \pm 4 \mu \mathrm{m}-35 \pm 7 \mu \mathrm{m}-58 \pm 12 \mu \mathrm{m}$

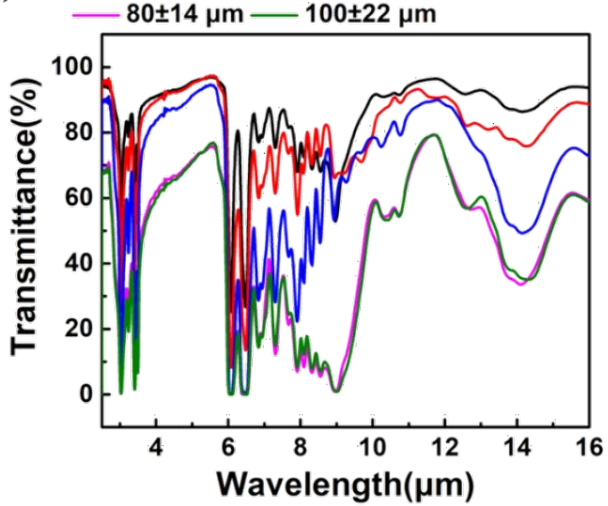

Figure S3. FTIR transmittance of $\mathrm{PA} 6 / \mathrm{SiO}_{2} \mathrm{NFM}$ with different a) mass ratio of $\mathrm{SiO}_{2}$ and $b$ ) pores size between nanofibers and c) thickness. 
(a)

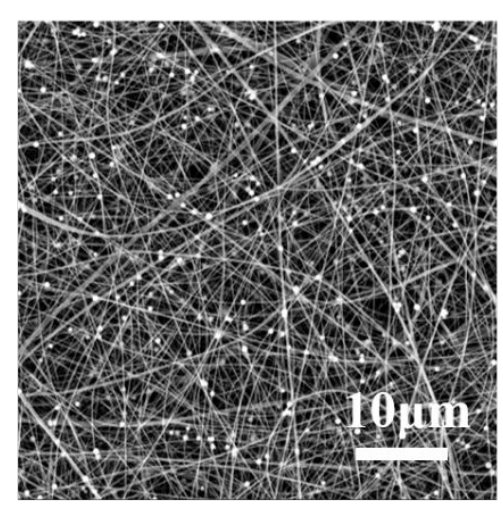

(c)
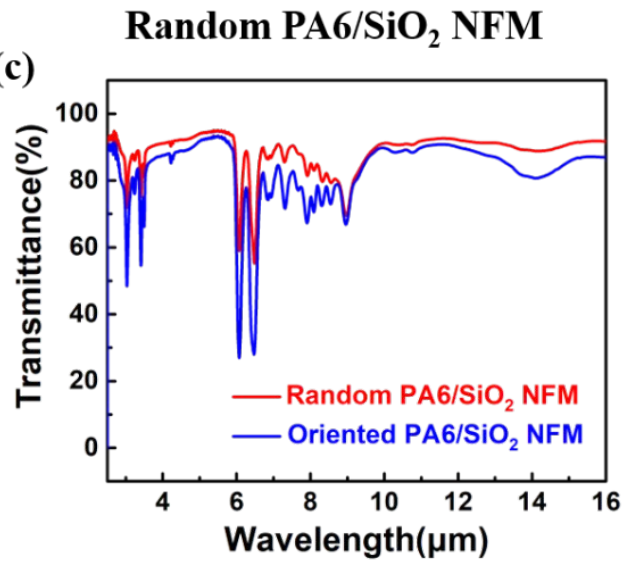

(b)

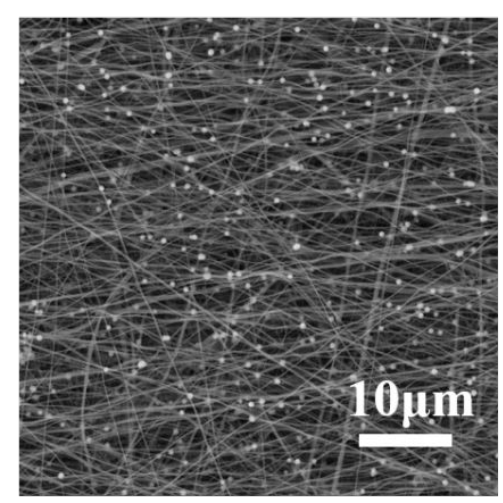

Oriented PA6/SiO $/ \mathrm{SFM}_{2}$

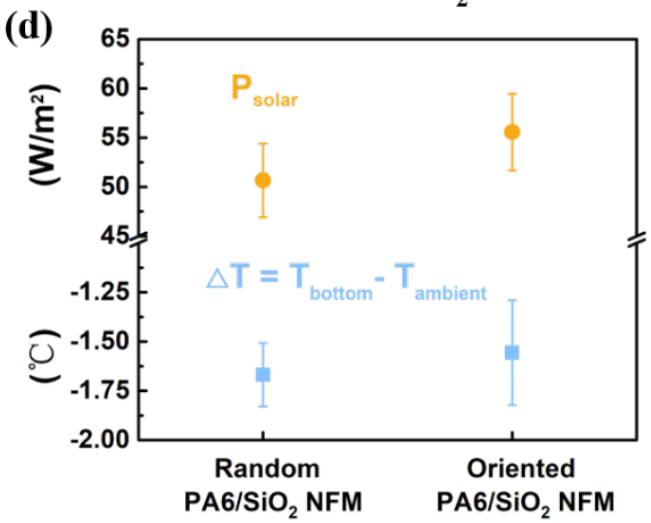

Figure S4. a, b)SEM images and c) FTIR transmittance and d) radiative cooling performance of random $\mathrm{PA} 6 / \mathrm{SiO}_{2} \mathrm{NFM}$ and oriented $\mathrm{PA} 6 / \mathrm{SiO}_{2} \mathrm{NFM}$. 


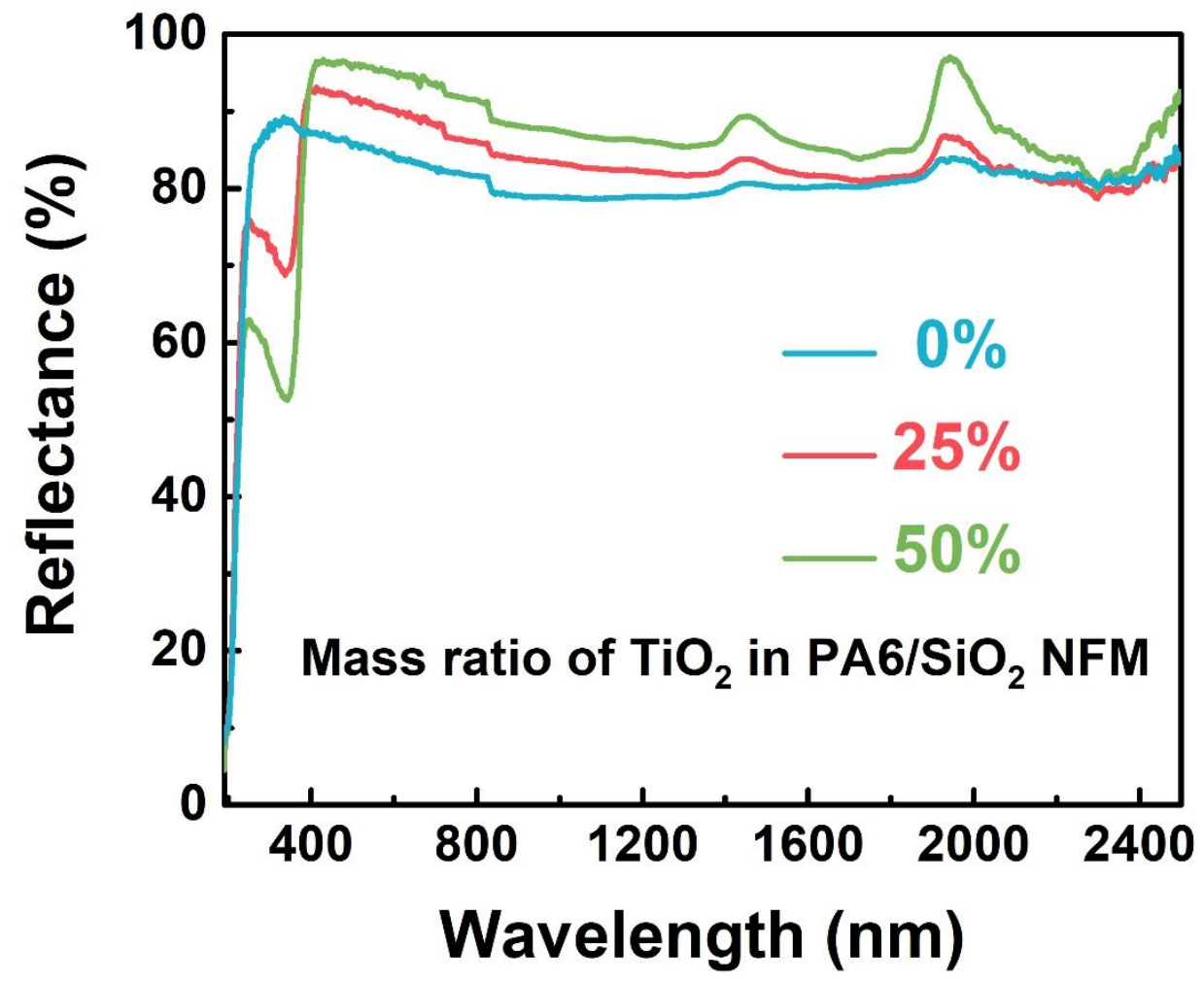

Figure S5. Reflectance of the $\mathrm{PA} 6 / \mathrm{SiO}_{2}$ NFM incorporated with different mass ratio of $\mathrm{TiO}_{2}$. 
(a)
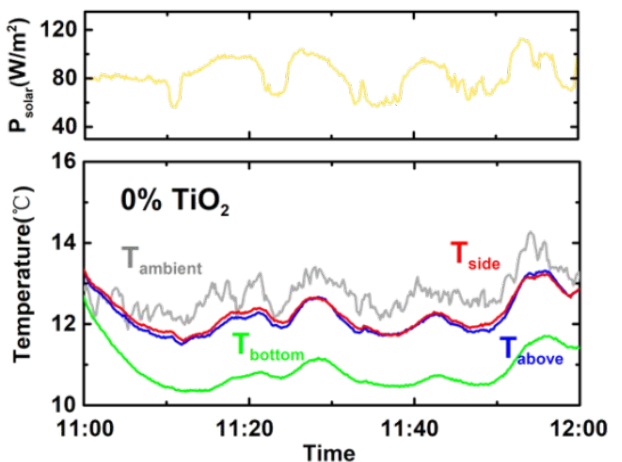

(c)

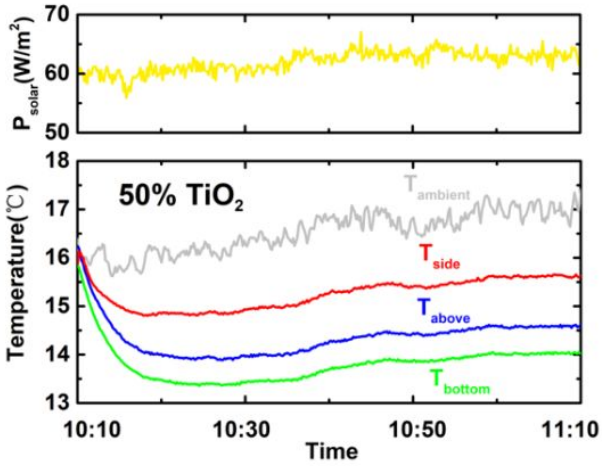

(b)
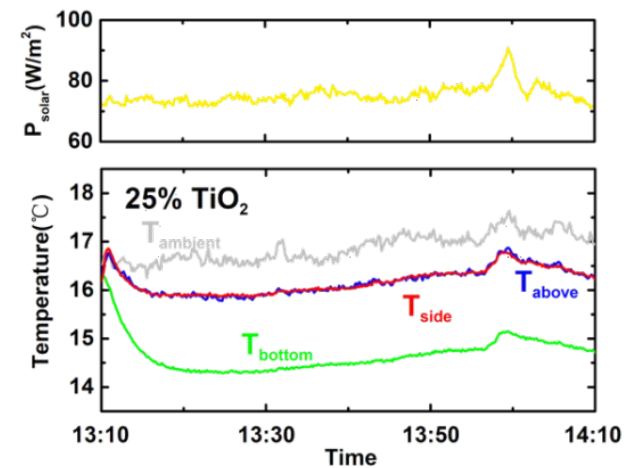

(d)

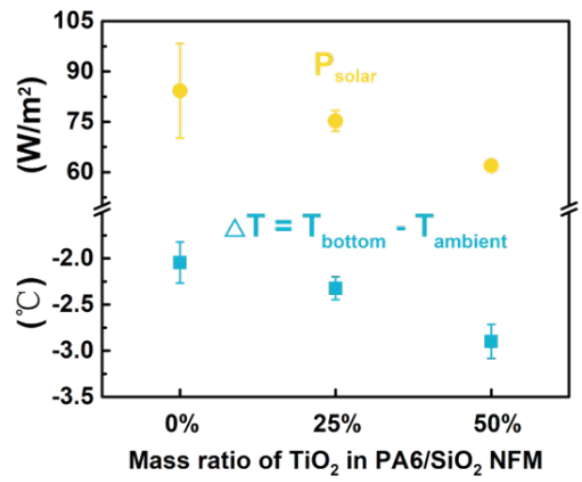

Figure S6. The cooling effect of $\mathrm{PA} 6 / \mathrm{SiO}_{2} \mathrm{NFM}$ incorporated with a) $0 \% \mathrm{TiO}_{2}$, b) $25 \%$ $\mathrm{TiO}_{2}$ and c) $50 \% \mathrm{TiO}_{2}$. d) the contrast of them (The error bars correspond to standard deviation caused by the statistical uncertainty of measurement). 


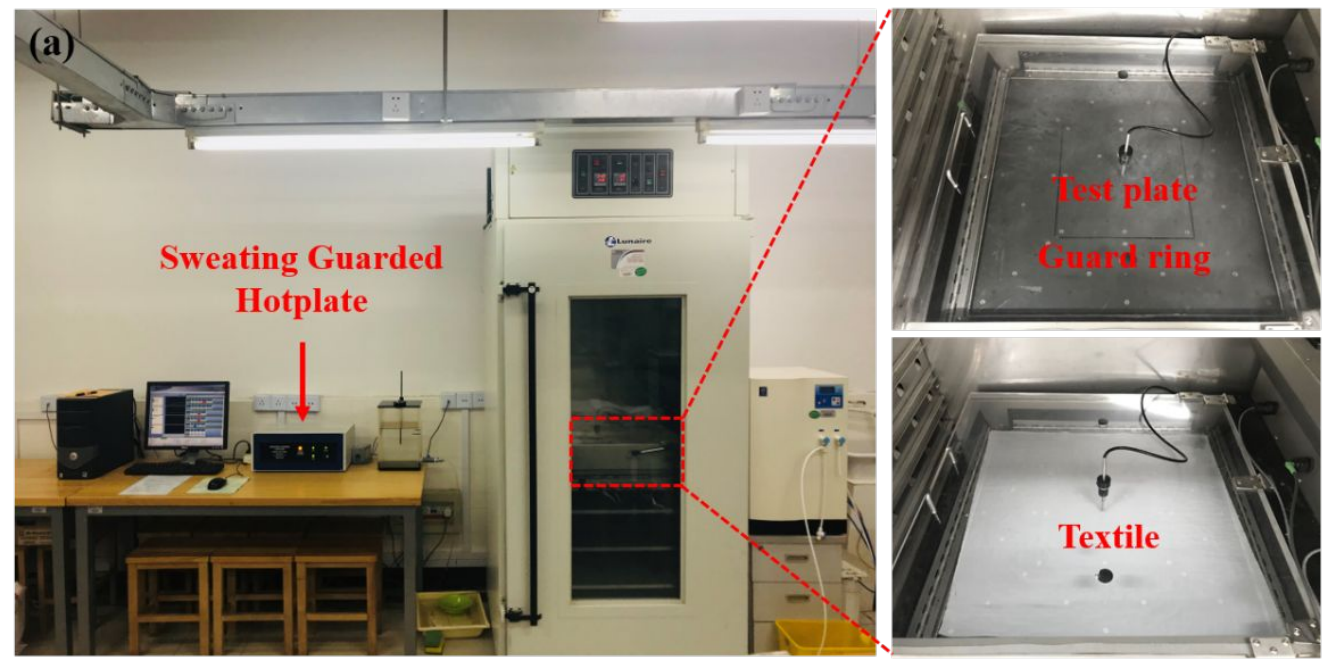

(b)

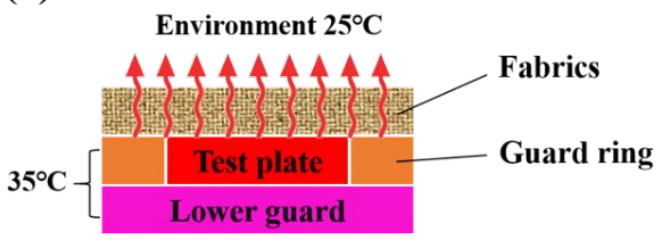

Thermal Resistance Test (c)

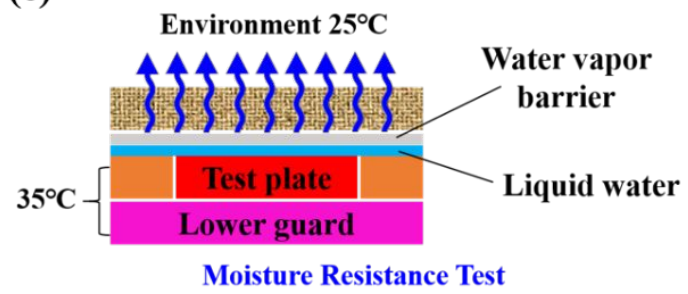

Figure S7. Photograph of thermal and moisture resistance test apparatus (a) and

Schematic of thermal resistance test (b) and moisture resistance test (c). 
(a)

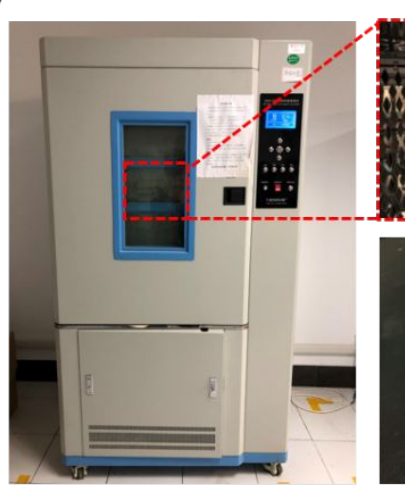

(c)

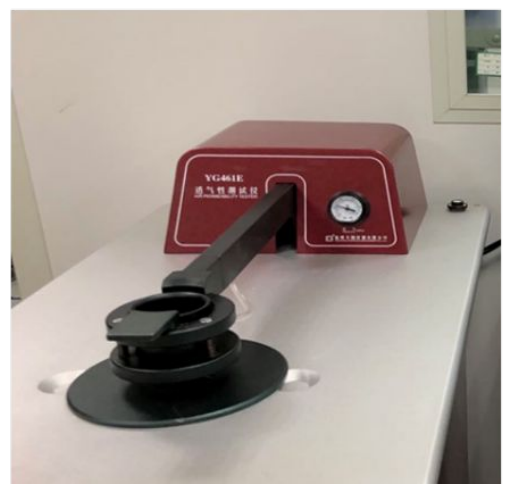

(b)

\section{T: $38^{\circ} \mathrm{C} \quad \mathrm{RH}: 90 \%$}

Moisture

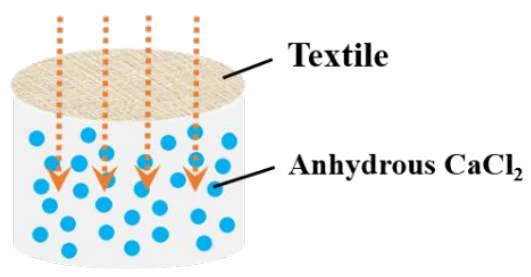

(d)

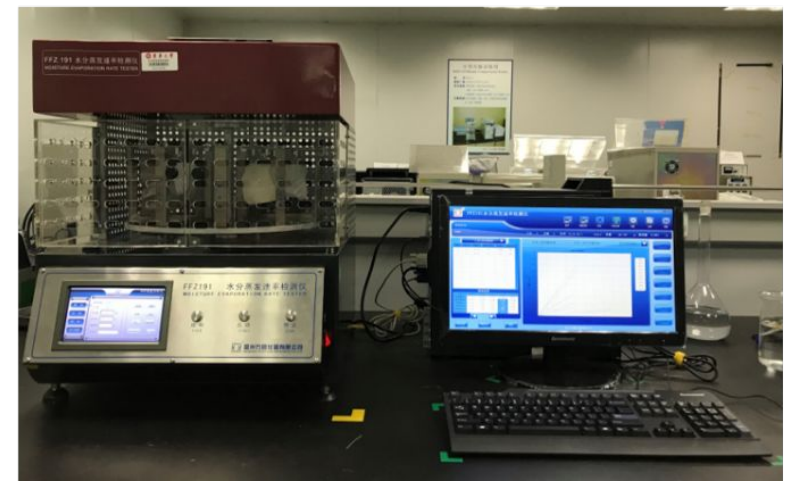

Figure S8. Photograph of a) water vapor transmission, c) air permeability and d)water evaporation rate test apparatus of textiles; b)Schematic of water vapor transmission test container at the environment of temperature $38^{\circ} \mathrm{C}$ and relative humidity $90 \%$. 
(a)

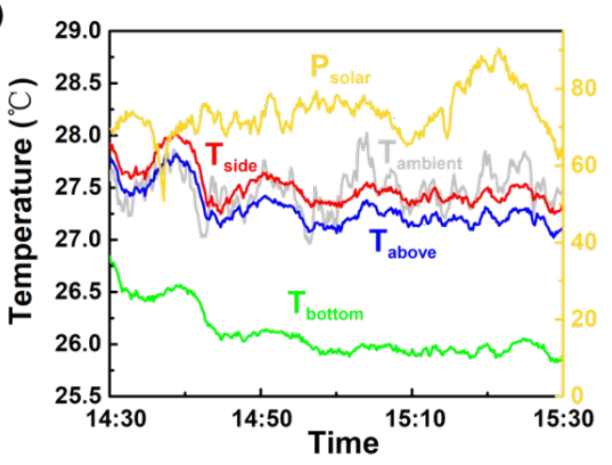

(c)

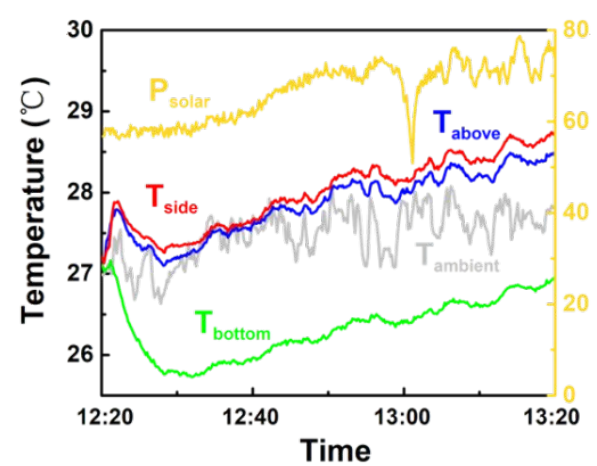

(b)

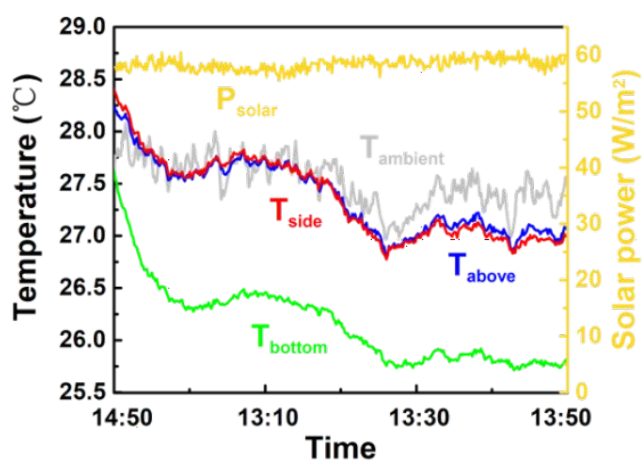

(d)

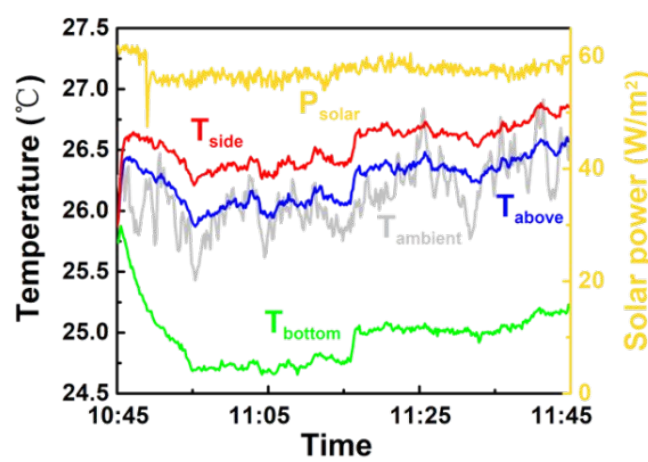

Figure S9. Radiative cooling performance test of the $\mathrm{PA} 6 / \mathrm{SiO}_{2} \mathrm{NFM}$ after washing for a) 0 , b) 1 , c) 5 , d) 10 times. 
(a)

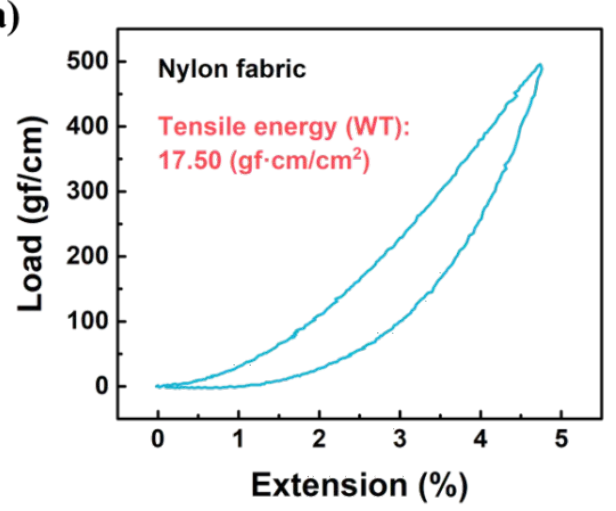

(c)

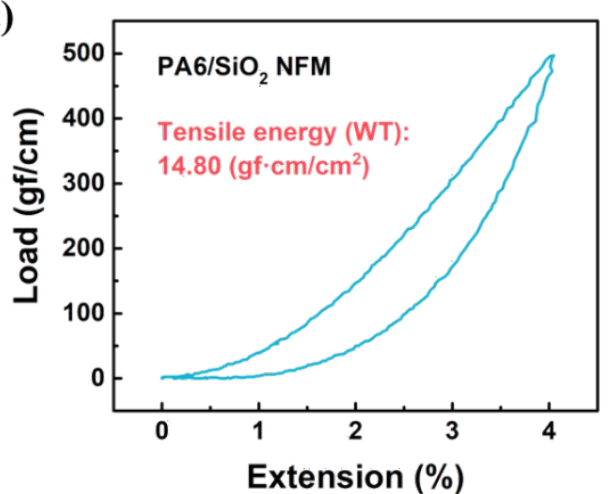

(b)

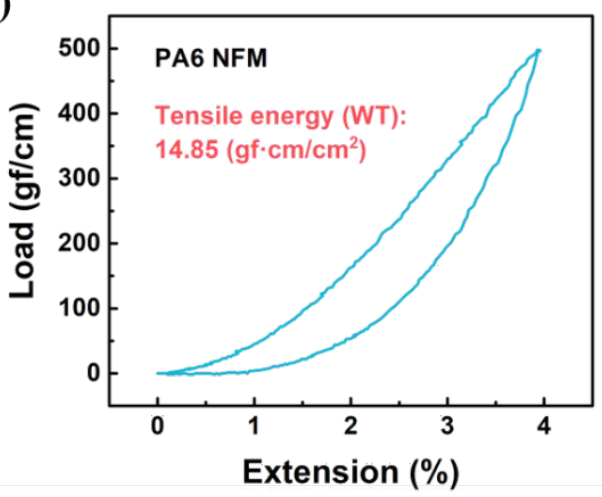

(d)

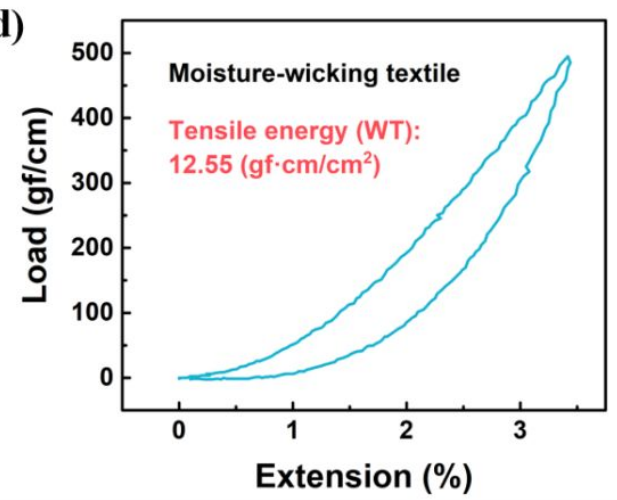

Figure S10. Tensile load (gf/cm) of a) nylon fabric, b) PA6 NFM, c) PA6/SiO $\mathrm{SFM}_{2}$ and $\mathrm{d})$ moisture-wicking $(\mathrm{M}-\mathrm{W})$ textiles as a function of extension $(\%)$. 